\title{
On Turbulence and its Effects on Aerodynamics of Flow through Turbine Stages
}

\author{
Galina Ilieva Ilieva
}

Additional information is available at the end of the chapter

http://dx.doi.org/10.5772/intechopen.68205

\begin{abstract}
In reality, the flows encountered in turbines are highly three-dimensional, viscous, turbulent, and often transonic. These complex flows will not yield to understanding or prediction of their behavior without the application of contemporary and strong modeling techniques, together with an adequate turbulence model, to reveal effects of turbulence phenomenon and its impact on flow past turbine blades. The discussion primarily targets the turbulence features and their impact on fluid dynamics; streaming of blades, and efficiency performance. Turbulence as a phenomenon, turbulence effects and the transition onset in turbine stages are discussed. Flow parameters distribution past turbine stages, approaches to turbulence modeling, and how turbulent effects change efficiency and require an innovative design, among others are presented. Furthermore, a comparison study regarding the application and availability of various turbulence models is fulfilled, showing that every aerodynamic effect, encountered of flow pass turbine blades can be predicted via different model. This work could be very helpful for researchers and engineers working on prediction of transition onset, turbulence effects, and their impact on the overall turbine performance.
\end{abstract}

Keywords: interaction effects, separation, transition, turbine blade, turbulence, vortices

\section{Introduction}

The phenomenon known as "turbulence" was already recognized as a distinct fluid behavior more than 500 years ago [1]. Figure 1 shows a sketch of L. Da Vinci, related to observations of free-stream turbulence.

Turbulence is defined as flow regime, characterized by changes in pressure and velocity, boundary layer separation, creation of vortex structures, and flow disturbances, etc. 


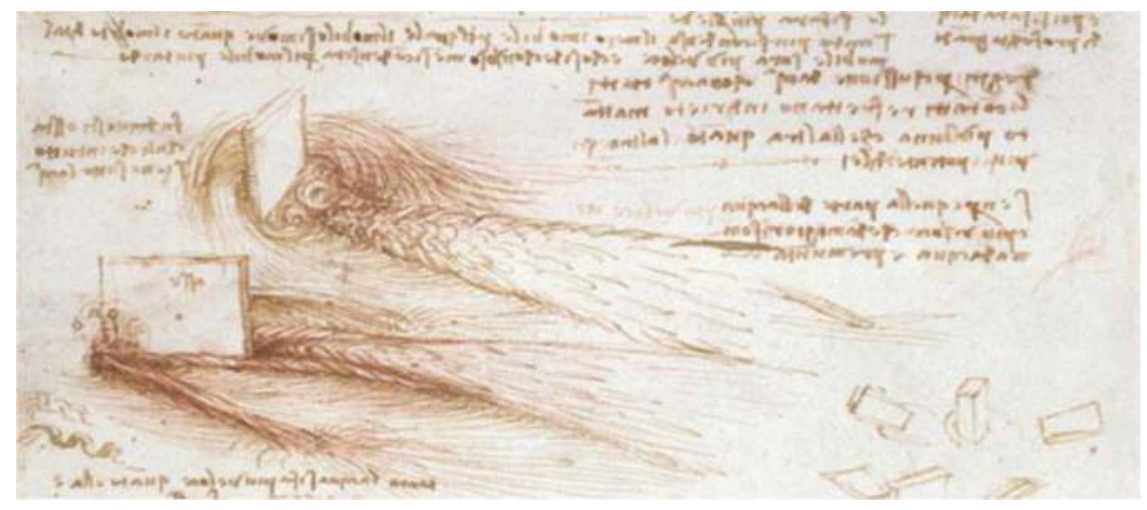

Figure 1. A sketch of turbulence by Leonardo Da Vinci, picture taken from [2].

Turbulence can be discovered in our everyday life and surrounding phenomena such as ocean waves, wind storms and smoke coming up from a chimney, among many others. Most flows observed in nature and existing in various machines and aggregates, such as pumps, fans, turbines, dryers, cyclones, stirred vessels, propulsion systems and their elements (propellers as an example), combustion chambers, and many others, are turbulent too.

In particular, a turbulent flow can be expected to exhibit all of the following specifics: disorganized, chaotic, seemingly random behavior; nonrepeatability in its structures; various and large range of length and timescales; enhanced mixing and dissipation, depending on the viscosity; three dimensionality, time dependence and rotationality; intermittency in both space and time [1].

Here, it is better to clarify that the so-called von Karman vortex street (behind a cylinder) is a vortex flow regular and coherent and cannot be referred to as a turbulent flow, Figure 2.

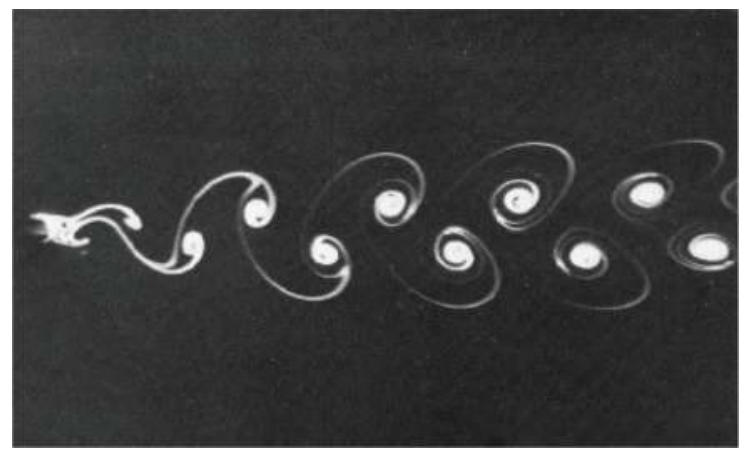

Figure 2. Von Karman streets behind a cylinder in a nonrotating $2 \mathrm{D}$ flow for $R e=140$, fluorescein visualization, picture taken from [3]. 
The onset of turbulence can be predicted by the Reynolds number, a dimensionless parameter; it characterizes the ratio between inertial and viscous forces. Reynolds number is equal to

$$
\operatorname{Re}=(\rho \cdot U \cdot L) / \mu,
$$

In this expression $\rho$ and $\mu$ are, respectively, fluid density $\left(\mathrm{kg} / \mathrm{m}^{3}\right)$ and dynamic viscosity (Pa.s), $U$ is flow velocity $(\mathrm{m} / \mathrm{s})$, and $L$ is length scale $(\mathrm{m})$.

When a subvolume of fluid is characterized by excessive kinetic energy, which is higher than the dampening effect of the fluid's viscosity, in other words, when very high Re number is realized in that subvolume, turbulence will appear. That is why turbulence is easier for observation in low viscosity fluids, but more difficult for observation in highly viscous fluids. If Reynolds is less than a definite critical value, damping friction forces prevent turbulent movement and the flow is laminar.

In a turbulent flow, vortex structures of various sizes and frequencies could be found. Large vortex structures, influenced by the domain boundaries and the global flow field, break up into smaller structures, characterized of higher frequencies. Small vortex structures are characterized by less frequency. However, due to the flow aerodynamic character and domain boundaries, small vortices can form bigger vortex structures and vice versa. At the same time, vortices in a volume, being at continuous interaction, can exchange energy among them, change their energy levels and travel in the flow volume, changing their so-called "mixing length."

Vortices, which contain the highest amount of kinetic energy, are described by the Taylor scale. In the inertial range, the vortex breakup can be described by inertial effects, thus viscous effects are negligible. Small vortices contain a low amount of energy but contribute mostly to the dissipation. The smallest turbulent vortices are defined by the Kolmogorov microscale [1].

In general terms, in turbulent flow, unsteady vortices appear of many sizes which interact with each other, exchanging energy, as a result drag increases due to friction effects.

The level of turbulence has significant impact on the stability of boundary and shear layers. High stream turbulence scales can contribute to earlier laminar-turbulent transition. Small turbulence scales, observed in the boundary layer, are very important for the skin friction levels and can exert the separation due to adverse pressure gradients. Thus, separated boundary layer and vortices lead to turbulent fluctuations and increase the level of unsteadiness. Shear layers, between the separated and main flow, act as cores of further developed turbulence levels and turbulent scales.

Turbulence of flow through turbine channels is a prerequisite to problems in blades' streaming; they lead to less levels of aerodynamic efficiency; changes in flow regimes; significant pressure fluctuations, causing vibrations; and variable forces acting on blade surfaces, among many others. Aforementioned, lead to worse efficiency, possible blades destruction, and problems related to other processes and elements, which make part of the turbine aggregate and installation.

The aim of this work is to discuss the complexity of flows through turbine passages with a particular emphasis on turbulence and its mechanisms and to explain their effects on turbine aerodynamics and efficiency. The chapter discusses some current state of the art in regard to 
modeling and prediction of turbulence features, adequacy of turbulence models to achieve physically correct picture for flow parameters distribution in turbine stages. The aim of this chapter is to discuss the follow characteristics and to provide the reader to a better understanding of turbulence mechanisms, their impact on other phenomena in turbines, on the design of turbine components and on the working regimes. Also, this work could be very helpful for researchers and engineers working on prediction of transition onset, turbulence effects, and their impact on the overall turbine performance.

\section{Turbulence and its relation to various processes and effects}

\subsection{Turbulence and interaction effects and losses}

Turbulence in turbine stages depends on many aerodynamic features and flow conditions. In this chapter, the author is trying to shed light, based on previously conducted researched works, on the unsteady effects and loss mechanisms in turbines, how they depend on the turbulence effects and how the aerodynamic performance could be compromised.

In [4], an extensive review of loss generating mechanisms in turbomachinery is presented. The three principal sources of losses in turbine stage, are described as: viscous shear in boundary layers, shear layers and mixing processes; nonequilibrium processes such as shock waves and heat transfer processes.

Boundary layers are known as highly viscous regions, also could be referred to as regions of steep velocity gradients and shear stresses. In the boundary layer, a higher amount of energy losses is produced in the areas where the steepest gradients are found [5].

In [6], a parameter called "dissipation coefficient " $\mathrm{Cd}$ is described. Its variation for laminar and turbulent boundary layers, with Reynolds numbers in the range of $300<\operatorname{Re} \theta<1000$, shows that the phenomenon of transition prediction is very important in the assessment of losses in turbomachinery boundary layers.

Stator and rotor blades interact, thus unsteady flow perturbations will appear both in stationary and rotating frames of reference. Various aerodynamic effects, such as wake shedding at the trailing edge, secondary flows in radial direction, blade vibrations, flow leakage in axial gaps, shock waves and effects at trailing edge in transonic stages, angles of attack at the leading edges, etc., can seriously affect the rotor blades and their efficiency performance. Blades loading and forces, acting on blades, are significantly lower under aforementioned conditions, leading to less efficiency [7].

Vortex shedding, as a phenomenon, will appear if flow detaches periodically from the back of a body, forming a Von Kármán vortex street. Such flow picture can occur at the trailing edge of the blade profile. The resulting frequency can be estimated based on the Strouhal number, Eq. (2).

$$
S t=(f e \cdot L) / v
$$


In Eq. (2) parameters are as follows: $L$ is the characteristic length in (m), the excitation frequency is $f e$, and $v$ is the flow velocity $(\mathrm{m} / \mathrm{s})$.

Turbulence intensity decays much slower than the velocity deficit in the wake [8]. The velocity deficit in an upstream blade wake can be perceived as an incidence variation by the downstream blade row. Turbulence levels in the wake can change the boundary layer from laminar to turbulent, leading to additional losses. The influence of wake will maintain through the blade rows far in a downstream direction.

Flow, characterized by low velocities, has normal velocity component toward the suction side of the downstream blade, thus an upstream wake with low velocities and higher turbulence levels can move toward the suction side of the blade. In a similar way, a fluid characterized by higher velocity can move toward the pressure side of the downstream blades. This movement of fluid particles has the following major effects on the downstream blade row, as described in [9]: change in boundary layer characteristics of a profile through its effect on the transition processes; affects the secondary flow generation through the blades in downstream direction; and influences the wake mixing losses due to the phenomenon of wake stretching or compression.

One of the first studies related to the interaction between streamwise vortices and downstream blades is described in [10]. A significant increase in random unsteadiness at the front part of rotor blades, in regions associated with stator secondary flow, was observed. This is a result of vortex breakdown, and is proposed that it occurred due the vortex filling and cutting, and the strong deformation of the vortex cross-sectional area, at the moment when the vortex enters into the rotor interblade channels [10]. The vortex energy is converted into energy of random fluctuations during the process of vortex breakdown. Later, more detailed mechanism for the vortex-rotor interaction, was developed, see Ref. [11]. According to that model, a moment after the vortex is separated from the rotor blade, thus disturbance will create and will start propagate along the vortex axis, at the local speed of sound, whilst simultaneously being swept downstream, at the local convection velocity. The place where vortex arises is located close to the pressure-side stagnation region [11]. In [12], for one and a half stage, it is demonstrated that flow passing through the first row of rotor blades is highly unsteady and is much influenced by the flow generated between two adjacent stator blades. Upstream, flow disturbances can convect through the rotor blades, without interaction with last mentioned, for a short time and vice versa [12].

In a passage of low aspect ratio blades, secondary flows generated in the form of streamwise vortices, are significant across the blade and can take more than 1/3 part of the blade span [13]. These vortices are convected downstream toward the next blade row where they interact with the main flow.

In [14], for first time, results of studies related to the origin and mechanisms of secondary flows generation, on the basis of the analytical modeling, Figure 3, are described. The incoming boundary layer is modeled as a vortex filament $\mathrm{ab}$ and is shown that when this filament convects in downstream blade rows to def, it produces three forms of vorticity: secondary, trailing filament, and trailing shed vorticity. The distributed secondary vorticity is result of 


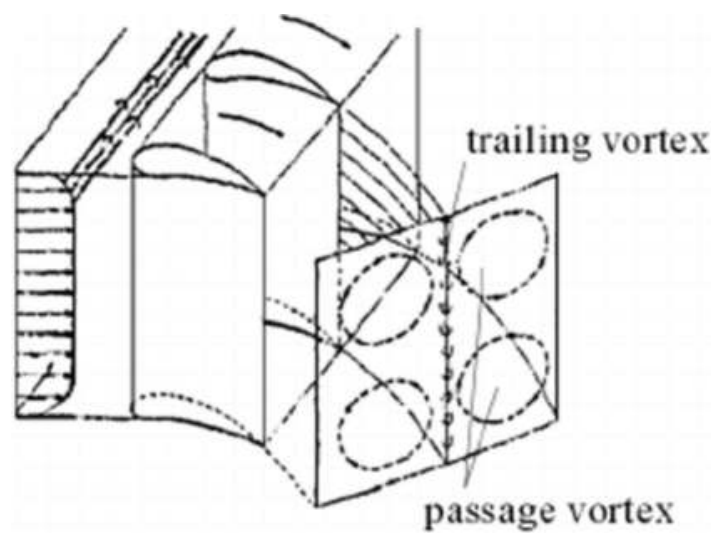

Figure 3. Model of Hawthorne for secondary flows, realized in 1955, picture taken from [14].

the turning of the inlet vortex filament; the trailing filament vorticity forms due to the differences in velocity between the concave and convex surfaces. The last one, the so-called trailing shed vorticity, is formed as a result of the spanwise variation of the blade circulation. Furthermore, it was stated that these mechanisms contribute to the overall secondary flow generation [14]. The Hawthorne's approach, applied and described in [13], is result of many assumptions; however, it can shed some light on the origin of the secondary flows. Similar models were developed and presented by other scientists $[15,16]$.

Every wake is initially represented as a perturbation in the uniform flow, as stated in [17, 18]. Wakes are transported with the main flow and are cut up into separate segments by the downstream blades. Inside the blade passage, the wake continues to behave as a jet, taking energy from the main flow. The velocity induced by that jet leads to generation of wake flow over the convex blade surface; the induced wake structure over the concave blade surface disappears. As a result of the blade circulation, in axial turbines, formed wake structures can stretch and shear over the blade surfaces or along the channel, they are travelling through [19].

Wake, before entering the blade passage, is subjected to "bowing" due to the higher velocities in the middle of the passage, in comparison to the near blade surfaces [20]. Moreover, wake experiences "shearing" near the suction surface and "stretching" near the pressure blade surface. It is due to the fact that the part adjacent to the convex blade surface convects more rapidly in comparison with the part adjacent to the concave surface. As a result of these processes of bowing, stretching, shearing, and distortion, the wake is moved to the convex blade surfaces and its tail is stretched in direction back to the leading edge of rotor blades. This wake transport is a prerequisite to losses generated from mixing of wakes in the downstream direction $[15,16,19,21,22]$.

Secondary flows lead to increase in losses, formed at the end walls, and to nonuniform distribution of exit flow angles. In turbomachines, the upstream end walls are rotating relatively to a blade row, thus the inlet boundary layer is expected to be skewed. Experiments, described in [23-25], show that already mentioned skew significantly can affect the existing passage 
vortices and losses. The streamwise vorticity, introduced to the flow, by the skewed boundary layer at the inlet, will strengthen the streamwise vorticity, observed at the exit; all that is visible in the direction of the turbine rotation.

Existing radial gaps lead to increase in the leakage mass flow rate and causes efficiency losses. Leakage flows are formed as a result of pressure differences between concave and convex blade surfaces, dominated by formed trailing vortices, shed in the downstream direction. These vortices can reduce the local turning, performed by the blade, and can generate decrease in the extracted mechanical energy. As a consequence of the viscous effects in the tip clearance, entropy increases. The second major feature is the subsequent mixing of flow, which passes through the tip clearance gap with that coming from the main flow. Flow structure in the tip region is studied and explained by a number of researchers; see Ref. [26-29].

Low pressure, generated immediately behind the trailing edge, leads to very high losses at the trailing edges. Flow expands around the trailing edge to that low pressure values and is then recompressed after a strong shock wave, where meet flows coming from suction and pressure sides [30,31]. The interaction between shocks and boundary layers can lead to unsteady boundary layer separations and increase in loss for transonic velocities [32, 33].

\subsection{Turbulence and condensation effects in two-phase flows in turbine stages}

In two-phase flows in turbine stages, the liquid phase could be presented both as water film, along the streamed blade walls, and as droplets, travelling with the main flow. The interface between primary and second phases is characterized by many aerodynamic and thermal effects.

Developed models for annular two-phase flows take into account differences and specifics for both the liquid and vapor phases and also introduce continuous and dispersed fields. Knowledge of the turbulence characteristics related to the continuous vapor phase, modified due to the presence of the droplet field, is required to introduce closure for those models. Vapor core turbulence is known to influence interfacial shear and affects transport and specifics in structure of the dispersed liquid droplet field, and ultimately interfacial shear effects and droplets dynamics and deposition are affected by vapor core turbulence.

- Condensation processes in turbomachines are random and unsteady phenomenon. Droplet growth rates show different characteristics, recognized in numerical simulations and experiments, also measured in real low-pressure steam turbines. These differences result by the large-scale temperature fluctuations, which are caused by the segmentation of blade wakes (mainly those at the trailing edges) by successive blade rows.

- In the case of two-phase flows, one has to clarify the effects of turbulence in relation to the gas and liquid phases. In other words, the gas phase turbulence can be affected in a two-way coupled manner by the dispersed phase and the droplet motion may also be influenced by turbulence. In case of liquid phase, the instantaneous flow velocity is decomposed into mean and fluctuating components: 


$$
\begin{gathered}
u_{i}=u_{i-}+u_{i}^{\prime} \\
u_{i}^{\prime}=\Pi(2 k / 3)^{1 / 2}
\end{gathered}
$$

where $\Pi$ provides normally distributed random number, which allows the fluctuating component $u i^{\prime}$ to take different values around a mean velocity $u i$.

The mean component of the fluid velocity will affect the average trajectory of flow particles. Two identical particles can have different trajectories due to the specifics in flow aerodynamics, such as secondary flows, turbulence, condensation, etc. In the case of turbulent two-phase flows, the fluctuating velocity component leads to particles' trajectory deviations.

Particles with $\operatorname{Re}<400$ tend to suppress turbulence and its effects; particles with $\operatorname{Re}>400$, enhance turbulence as a result of vortex shedding [34].

In [35] turbulence measurements in flows with liquid film interface are reported; it was shown that turbulence intensities were higher than those in a pipe with wall roughness equivalent to that of the film interface.

Aerodynamic and thermal losses in turbine stages, due to the presence of second phase and turbulence effects, are as follows:

- Water film losses, originating from the movement of the water film on the blade surfaces;

- Drag loss due to the presence of coarse water droplets: small fraction of the overall losses originates from acceleration of the large coarse droplets at the trailing edges;

- Coarse water braking losses: kinetic energy of droplets decreases by impinging on the following blade row;

- Fog droplet loss due to the interphase velocity slip, entropy increases due to the viscous drag;

- Fog droplet deposition or collection loss, turbine efficiency performance is affected by decreased kinetic energy of fog droplets, merged on the blade surfaces.

The influence of direct losses in nonequilibrium flows grows with the number of stages and reaches its maximum at the end of the steam turbine. The indirect losses cause changes in aerodynamic loss by departures from equilibrium.

- Changes in flow-incidence angle, leading to increased losses, especially at the tip regions with thin leading edge blade profiles;

- Changes in profile losses due to the variations in surface shear stress, leading to transition and separation;

- Interactions between both the condensation-induced shock waves and formed boundary layer;

- Nonuniformity effects-pressure changes along streamed profiles change the working conditions for the downstream blade row. 
Other specifics of the two-phase flow pass turbine surfaces are as follows:

- Formation of water film over streamed surfaces and its defragmentation due to acting viscous forces, drag forces, pressure differences, secondary flows, deviation of streamlines, water particles, trajectories of water particles hit blade surfaces;

- Depending on the axial gap, deviations of streamlines, rate of condensation and water content and specifics of pressure and velocity distribution, weaker and stronger turbulent effects are observed;

- Boundary layer separation much before the trailing edge is attained, leads to formation of droplets of various diameters, most of them are condensation nuclei in the process of blades streaming by two-phase flow [36].

From structural point of view, coarse droplets together with the turbulence impact blade surfaces, leading to erosion and efficiency decrease [36].

\subsection{Turbulence and cooling in gas turbine passages}

Turbine blades, as part of gas turbine aggregates, must be cooled as they are highly exerted by temperature loads, deformations and stresses. Furthermore, unsteady effects, rotationally induced forces (Corriolis and centrifugal forces), and secondary flows and change in flow parameters, among others complicate the flow aerodynamics within the interblade channels.

The flow field is characterized by higher levels of turbulence, transition effects, secondary flows, unsteadiness, particularly due to the cooling flows and effects. Turbulence results due to wakes from upstream stages; rotationally induced forces; cooling fluid (coolant) that mixes with the main stream in turbine channels; interaction effects between film cooling jets and the mainstream flow; complex flow conditions from the combustor; separation of cooling film, separation effects associated with cooling jets and cavities, etc.

Heat transfer coefficients increase by the enhancement of flow turbulence levels and boundary layer separation effects. Last mentioned are accompanied by increase in the examined pressure drop [37].

The effect of turbulence levels on heat transfer, aerodynamic performance, drag forces, skin friction and flow parameters distribution has been studied by many researchers in last decades [38-42].

High turbulence levels are provoked due to the presence of upstream wakes and rotation effects [43-45]. High levels of turbulence lead to increased temperatures over blade surfaces, particularly at leading edges where temperatures reach maximum values [46].

As an effect of the interaction between cross-flow and film cooling, higher turbulence levels present in turbine flows. These effects depend on the diameter of holes that supply the coolant jets and their exact place on turbine blade surfaces. Experiments on cooling via inclined holes, characterized by ratio $(\delta 1 / d<<1)$, show that a higher percent of turbulence levels can be achieved immediately in the downstream direction of a row of film cooling holes [47]. Turbulence effects and their increase are found even after film cooling jet dissipation. 
By application of hot-wire measurement techniques is also concluded that in the case of $(\delta 1 / d<<11)$ jet-turbulent fluid could dominate the boundary layer in the downstream direction [48].

In [49], authors have studied how circular wall jets can increase the free-stream turbulence intensity. It is found that the increased turbulence levels in the free-stream can cause high levels of mixing and quick dissipation in the film layer.

An increase in heat transfer levels is related to moderate turbulence intensity levels and relatively small length scales recognized in the fluid.

Mainstream turbulence intensity and length scales have significant impact on the film cooling jets and their intensity in the flow, also lead to additional interactions between cooling jets and core flow and affect the effectiveness of film cooling in the downstream direction [50].

Free-stream turbulence can decrease the efficiency of film cooling, in the downstream direction and can increase it between injection holes, due to the enhanced mixing [51].

An increase in mixing of momentum, that effectively thins the boundary layer, will result in an increase in the film cooling efficiency in the downstream direction. At the same time, an increase in the rate of cooling film disintegration is a reason for less efficiency performance of the film cooling. In general, which case will be dominant depends on the "blowing ratio" [37, 52]. In [53-55], the effects of increased turbulence intensity on film cooling performance, at leading edge were examined and the effects of increased turbulence intensity are in a strong relation to the blowing ratio.

The efficiency of performed film cooling varies rapidly with the blowing ratio and is in function of the spanwise angle [56].

At higher blowing ratios and cylindrical film cooling holes, the effectiveness in downstream direction will increase if the jet angle is deflected in a close proximity to the wall.

An increase in turbulent mixing levels, due to free-stream turbulence, leads to improved spanwise mixing between holes and better film cooling performance [57].

Film layers characterized with low blowing ratios could be rapidly dispersed by increased turbulence intensity, while increasing the local heat transfer coefficient. High blowing ratio film layers were found to be relatively insensitive to increase in the turbulence intensity levels, consistent with previous studies with flat plates.

Many numerical and experimental studies have been conducted to determine the relation between turbulence and the Nusselt number. A numerical study of both heat transfer characteristics and flow field of a slot turbulent jet impinging, on a concave surface with constant heat flux, has been carried out and discussed in [58]. In [59] attention is paid on flat plate with varying curvature, results are discussed in [59]. Computational results show that maximum value of $\mathrm{Nu}$ is attained at the stagnation point and gains higher values as the Reynolds number increases. 


\section{Transition modeling in turbine stages}

\subsection{Transition as a phenomenon: transition in turbine stages}

Turbulence modeling has been a subject of intensive research for many years. Turbulent models are able to predict turbulence occurrence and its effects in turbine passages. However, in the case of modeling, with all specific features, such as stator-rotor interaction, phase changes (if any), compressible and viscosity effects, pressure gradients, among others, turbulent model lacks of applicability is not able to provide accurate predictions of turbulence effects on the mean flow characteristics. Models should be more powerful and enriched by specific terms related to existing specific aerodynamic observations due to turbulent effects.

One of the most important and most difficult turbulent phenomenon, to model and resolve, is the so-called transition.

Reynolds is the first scientist who worked on the transition phenomenon; he investigated the transition from laminar to turbulent flow by injecting a dye streak into a flow through a pipe having smooth transparent walls [1].

Prediction of the onset of boundary layer transition is one of the most important concerns in the area of fluid mechanics. There is a great interest to transition as it plays a major role in many engineering applications and raises important questions to the flow physics, also could serve as an ingesting example for determinism and chaos.

The so-called viscous instability of a laminar boundary layer was for the first time taken into account and studied by Tollmien. Under low free-stream turbulence conditions, instability is observed in the case of two-dimensional unstable Tollmien-Schlichting waves are formed and propagate in the streamwise direction. These waves lead to additional $3 \mathrm{D}$ aerodynamic effects to appear in the flow structure, such as peaks, stronger secondary flow effects, hairpin vortices and transition effects. Turbulent spots are formed in the regions of vorticity peaks and can develop to continuously spreading turbulence. A turbulent spot model to describe the specifics of a transitional flow is proposed in [60]. Later, turbulent spots generated over a flat plate surfaces, without imposed pressure gradients, were also visualized [61]. Recently, scientists have been working on more accurate transition length predictions, based on measurement of transition length in a field of adverse pressure gradients and of triggered turbulent spots, see Ref. [62]. It was found that spot characteristics, in the case of adverse pressure gradients, are different from those formed in the case of zero or favorable pressure gradients. Also, it became clear that in the presence of adverse pressure gradient, a spot can be formed at the center of a highly amplified transverse waves and is convected at lower velocity than under a zero pressure gradient, as discussed in [63].

Laminar to turbulent transition is proved as a phenomenon, which seriously affects the efficiency performance of various machines. The transition effects contribute to additional drag and lift forces, also heat fluxes that are crucial for overall working principles of different types of machines and installations. 
A particular field, in which there is a specific interest to the transition phenomenon and its physics, is the area of turbomachinery. Transitional flows can be seen in flows past turbine blades, mainly in low pressure turbines. Transition is observed when various geometry blades are streamed at variable flow parameters and boundary conditions. Turbulence and transition effects significantly decrease the aerodynamic performance of turbine stages and must be studied and understood in detail [64].

Speaking about transition, one must stress that there are different types of transition [65]. The first one is called "natural" transition. It begins with a weak instability in the laminar boundary layer, as was described years ago by Tollmien and Schlichting [6], next proceeds through various stages of amplified instability to fully turbulent flow. The second type of transition is the "bypass"" transition, defined by Morkovin [84]. Bypass transition is caused by high levels of disturbances in the external flow (such as free-stream turbulence) and can completely bypass "natural transition." The third type of transition is the so called "separated-flow" transition. It exists in a separated laminar boundary layer and may or may not reveals some instabilities of the Tollmien-Schlichting type.

As it is related to the "bypass" transition, some researchers presumed that it could appear as an instantaneous turbulent breakdown with zero length of transitional flow. However, the bypass transition does not always exclude instability processes. Only the long region of twodimensional wave amplification preceding the appearance of three-dimensional disturbances (spanwise periodicity) in low turbulence flow is bypassed, as clarified in detail in [63, 66]. In more detail, during the phase of the so-called "bypass transition," not all specific laminar breakdown processes would be recognized. Separated-flow transition occurs when a laminar boundary layer separates and transitions in the free shear layer, above the already formed bubble. Transition due to separated flow could develop at the leading edge and close to the place where minimum pressure on the suction surface sides is formed. This type of transition is extremely harmful for low pressure turbines and leads to early separation of the boundary layer. There is another type of transition, reverse transition, which is known as "relaminarization." Relaminarization is possible to appear at places where a previously turbulent or transitional region is affected by strong favorable pressure gradients, and as a result of that, it transfers to laminar again. Depending on the profile section geometry and the flow regime, near the leading edge, laminar flow followed by a wake-induced or shock-induced transition could be visualized. Last described phenomenon could be replaced by a relaminarization with subsequent transition to turbulence, occurring at multiple locations simultaneously [63].

The phenomenon of "separated-flow transition" could arise after the so-called boundary layer trip wires as a result of laminar separation under strong adverse pressure gradients. Thus, the flow can reattach as turbulent, forming laminar separation/turbulent-reattachment "bubble", on the surface under consideration. In gas turbine stages, the transition of separated flow could be seen in the so-called overspeed region close to the leading edge of the profile, over the convex or concave side, or both, and near the place where minimum pressure on the convex side is observed. What will be the bubble size depends on the transition process within the free shear layer and may involve all of the stages for a natural transition type. For bubbles with bigger size and characterized by low free-stream turbulence levels, flow in the 
bubble is dominantly laminar and instabilities could be observed [67]. Big bubbles, along the blade surfaces, produce losses and act as a prerequisite to exit flow angles deviation. Small bubble configurations are an effective way to increase the turbulence levels and can possibly control the blade aerodynamics [63].

Speaking about transition and its effects on the entire flow field, it is necessary to mention that flow passing through a turbine stage is essentially turbulent and unsteady. The nonstationary pulsations are obtained as a result from the stator-rotor interaction, mainly. Periodic phenomenon, caused by stator-rotor interaction effects, excites both the flow, passing over blade surfaces, and boundary layer characteristics [63]. This results in an increased production of the so-called turbulent spots and shifts the location of laminar-turbulent transition in the upstream direction. This laminar-turbulent transition phenomenon is known as "wake-induced transition" [65].

Figure 4, a picture of possible boundary layer development over surfaces of high pressure blade is shown, see [65]. On the suction side, it is usually expected that in the downstream direction of the initial laminar part, a boundary layer will transfer to turbulent (2) in Figure 4. The size of the transition zone is related to the place where transition phenomenon could be observed - in upstream direction or downstream direction of the place of minimum pressure. In the upstream direction, the zone of transition is expected to comprehend bigger area. If a laminar separation bubble occurs in the front part of the suction side (1), then the presence of high pressure gradients will force the boundary layer to develop as laminar again in downstream direction; forward transition will take place [63]. The reverse transition may appear on the suction surface [67-69]. In the case of research on film-cooled gas turbine blades, the transition is expected to appear at the places where cooling jets are injected in the main flow [63]. In downstream, a reverse transition process also could be recognized. This fact could affect the heat transfer distribution over surfaces of film-cooled blades. On the profile pressure side - if a separation bubble occurs, the reattached turbulent boundary layer may become again laminar like, (2) in Figure 4. In the case of lack of separation bubble, a forward transition zone, followed by a reverse one, in the rear part of the profile, could be observed, (1) in Figure 4.

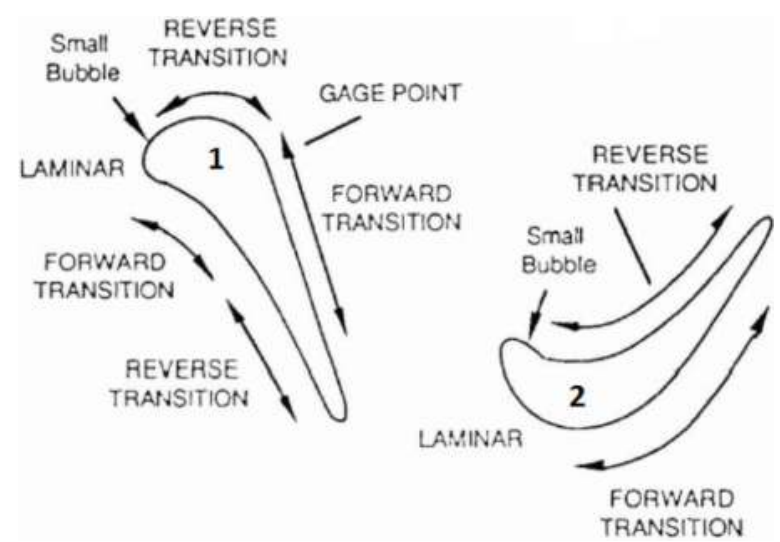

Figure 4. Boundary layer development on high pressure turbine blades [65]. 
In high pressure turbines, the effect of transition on losses is usually small, because the aerodynamic losses are mainly related to the turbulent flow development after the moment of transition. In low pressure turbines, the flow in interblade channels is characterized by low $R e$. Especially for gas turbines, as part of aircraft engines, the operating Reynolds numbers are low at high altitudes to begin with and a further decrease can cause separation before transition.

In regions where expansion occurs, the fluid is highly accelerated and the boundary layer has small thickness due to the favorable pressure gradients.

At high Reynolds numbers, transition occurs far in the upstream direction, flow is mainly turbulent over the profile. Near the trailing edge, in function of the blade profile geometry, the boundary layer will separate forced by turbulent levels. When Re number decreases, turbulent separation disappears and transition (the "bypass transition") moves in the downstream direction; at that moment losses are minimal. If Re number decreases more, laminar separation ahead of the transition region could appear. In the case of no separation, the bubble is small enough so that the flow could reattach to the blade surface. In this case, aerodynamical losses are slightly higher than the previously described case. For lower Reynolds numbers the increase of laminar shear layer and transition length, until reattachment, before the trailing edge, is no longer possible and thus a complete separation occurs, Figure 5.

\subsection{What transition depends on?}

In general, the turbulence Reynolds number $\left(R e_{\theta t}\right)$ increases with increase in acceleration or with decrease in the free-stream turbulence levels. The effect of acceleration is significant for low turbulence levels. However, for turbulence levels found in gas turbine stages, it has a negligible value. In the case of high turbulence levels, the transition onset is controlled by the free-stream turbulence [65].

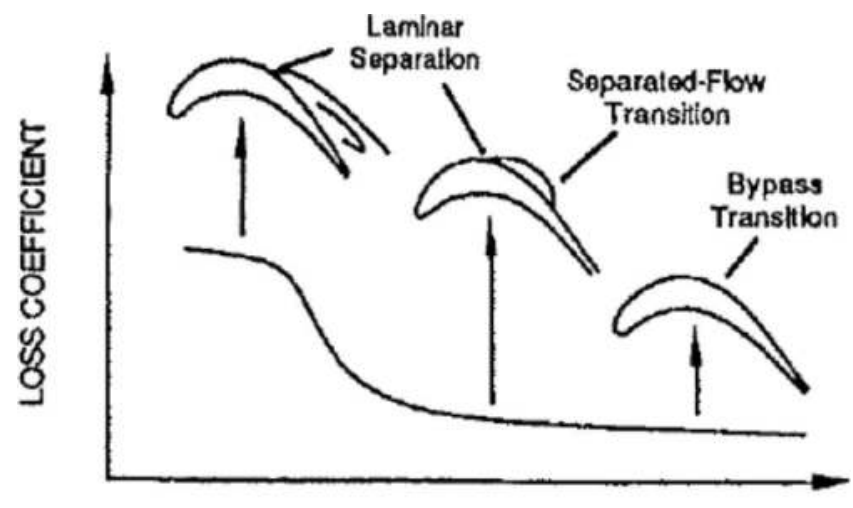

REYNOLOS NUMBER, In $\langle R \theta\}$

Figure 5. Transition on a low-pressure turbine airfoil at various Reynolds number. 
For accelerating flows, length of transition is variable for thermal and momentum boundary layers $[70,71]$.

The local point where boundary layer can separate is not in a close relation to the levels of free-stream turbulence. In the case of observed increase in levels of free-stream turbulence, the separation bubble size decreases and turbulent transition point moves in upstream in regard to the flow direction. The separation bubble decreases with increase of the Reynolds number [72]. At high free-stream turbulence intensity, the streak structures can be observed in the upstream direction and are related to the place of boundary layer separation, showing that bypass transition of an attached boundary layer can be realized at the high Reynolds number. In the case of low free-stream turbulence intensity, velocity fluctuations are seen within the shear layer of the separation bubble.

The roughness over streamed surfaces is nonuniform and characterized by significant variations in streamwise and spanwise directions [73, 74]. Modifications in the behavior of the boundary layer due to the presence of definite values of surface roughness can decrease the aerodynamic efficiency [75-77] and can also increase the heat transfer [78-80]). Levels of heat transfer may also be affected by changes in material properties or in the case of eroded or broken protective coatings.

The effect of geometry curvature and/or streamlines curvature on the transition was studied and described in [81, 82]. In [81], it was found that a laminar boundary layer formed over a concave surface becomes unstable as a result of acting centrifugal forces, three-dimensional disturbances and streamwise vortices presented in the boundary layer. Liepmann in [82] showed that transition on a convex surface is only slightly delayed, but can occur earlier over the concave surface.

The increase in transition Reynolds number is caused by the Görtler vortices, which can increase velocity gradients near the wall and thus can delay the transition. For highly curved surfaces, this effect dominates that one caused by turbulence. A concave curvature can decrease or can increase the transition The Reynolds number depends on the turbulence intensity and the curvature.

Heating or cooling can seriously affect boundary layer transition at low free-stream turbulence. Heat transfer through a laminar boundary layer formed over the concave blade surface is influenced both by Taylor-Görtler vortices and the main flow turbulence levels [83]. Transition occurs when these factors surpass the tendency of boundary layer to remain laminar in the presence of higher pressure gradients. If spot production is not affected by the heat transfer, at high free-stream turbulence intensities, transition would not be observed.

Film cooling affects the boundary layer formed on the streamed surfaces of gas turbine blades. At places where cooling fluid is injected, holes are usually much larger than the boundary layer thickness, thus the injection of coolant through these holes disrupts the flow close to the surfaces and provides higher turbulence levels within the downstream developing boundary layer [63]. Therefore, it may be said that film cooling effect is to "trip" a laminar boundary layer and initiates transition to turbulence. 
In the case of acceleration, sufficient to cause reverse transition in the downstream direction of the injection, the heat transfer intensity approaches that for laminar flow [69]. This implies that even though injection can initiate transition, a subsequent strong acceleration can cause the flow to become laminar again. Such a situation is common for film-cooled blades of first gas turbine stages. Heat transfer measurements on a stator vane, presented in $[85,86]$, indicated that the behavior of the boundary layer transition along the suction side of the vane showed dependency to the film-cooling injection place.

\section{Numerical modeling of turbulence in turbine stages}

Many numerical and experimental research works have been performed, and various codes and approaches have been developed and applied, for the purposes of modeling and research of origin and mechanisms of laminar-turbulent transition and how it exerts the fluid dynamics in turbine stages. However, the phenomenon in turbomachinery flows is not well understood. Transition modeling still limits the performance of nowadays CFD codes, and problems in estimation of the transition onset and extension of the transition affect the efficiency by several percent and the component life by more than an order of magnitude [87, 88]. Transition as a phenomenon and its understanding is of huge importance to arrive to appropriate design of blade geometry and increased aerodynamic performance.

A brief history of development of turbulence models dates back more than 140 years ago and is shown in [89].

Many of existing turbulence models are applied for modeling and research of turbulence effects in turbine stages. The performances of standard $k-\varepsilon$ model, RNG $k-\varepsilon$ model, realizable $k-\varepsilon$ model, SST $k-\omega$ model, and LRR Reynolds stress transport models for the purposes of research on the convective heat transfer, during slot jet impingement over flat and concave cylindrical surfaces, were evaluated against available experimental data [90, 91]). Near-wall models such as equilibrium wall function and two-layer enhanced wall treatment were applied to the boundary layer to obtain physically correct results. When the impingement surface is within the potential core of the jet, applied models overpredict the Nusselt number in the impingement region, and at the same time, $N u$ is not very correct in the wall jet region. The RNG $k-\varepsilon$ model, applied together with the enhanced wall treatment, also the SST $k-\omega$ model, gives better Nusselt number distribution in comparison with other models for flat plate and concave surface impingement cases. However, mean velocity profiles are not well predicted by the SST $k-\omega$ model for the concave surface impingement case. Results for velocity profiles, obtained with RNG $k-\varepsilon$ model, agree very well with the experiment. The Reynolds stress model could not give better prediction, compared to other eddy viscosity models [91].

In $[58,90]$, performance of various turbulence models to predict the convective heat transfer for slot jets impinged on flat and concave surfaces was under consideration. The outcomes as a result of application of more specific model are found very accurate in the case of an impingement surface placed outside of the jet core. In the case of surface placed inside the jet 
core, results obtained for $\mathrm{N} u$ demonstrated larger discrepancies and variation in the impingement region, as their models overpredicted the $N u$ in that region. However, prediction of values for $\mathrm{Nu}$ is fairly accurate in the wall jet region.

Other interesting numerical studies on rib-roughened channels are related to measurements and simulations with standard $k-\varepsilon$ and nonlinear $k-\varepsilon$ turbulence models [92].

The predicted Nusselt number depends on the selected turbulence model. An improvement in the predicted Nusselt number was found when comparing the LES with a standard $k-\varepsilon$ turbulence model [93].

The standard $k-\varepsilon$ and RNG $k-\varepsilon$ models can accurately predict streaming over an impingement surface [94].

As a result of the numerical modeling and analysis on turbulent flow field and heat transfer in 3D ribbed ducts, it is found that heat transfer predictions obtained using the $v^{2}-f$ cannot cover well the experimental data for the $3 \mathrm{D}$ ribbed duct. On the wall of the duct where ribs exist, predicted heat transfer agrees well with the experimental data for all configurations, heat transfer predictions on the smooth-side wall do not cover well the experimental data. It is mainly due to the presence of strong secondary flow structures which might not be properly simulated with turbulence models based on eddy viscosity.

The standard $k-\varepsilon$ turbulence model with wall functions cannot predict very well heat transfer, due to the presence of large separation regions in the flow field [95]. In [96], it was found that the $k-\varepsilon$ model can give only reasonable qualitative agreement with the experimental data. The application of standard $k-\varepsilon$ model to complex 3D problems is computationally expensive and leads to wrong results $[97,98]$. Application of two-layer $k-\varepsilon$ with the effective viscosity model gives bad predictions for heat transfer in rotating ribbed passages [99]. Computations with low-Re models could give good heat transfer predictions by introducing a differential version of the Yap length scale correction term [100].

Since low-Re models cannot correctly capture the separation and reattachment that takes place between ribs, $v^{2}-f$ and Spalart-Allmaras (S-A) were taken under consideration in many research works. The $v^{2}-f$ turbulence model was successfully applied to separated flow in [100], to 3D boundary layers [101], impinging jets, [102, 103] and prediction of flow characteristics and heat transfer in 3D duct with ribs and in model configuration, resembling the tip of an axial turbine blade [104].

The model of Spalart and Allmaras [105] was proven to be robust and accurate in aerodynamic applications [106].

As discussed before, the phenomenon of transition is very complicated and depends on many parameters, such as free-stream turbulence, roughness, curvature, heat transfer and film cooling, among others, and needs specific mathematical models and turbulence closures for the purposes of its research and analysis.

Due to the strong accelerations and decelerations of flow in turbine cascades, the local value of free-stream turbulence, at the location of boundary layer transition onset, may significantly 
vary from first to the last turbine stage. Currently applied transition onset correlations involve data from many scientists, who have adopted different approaches to define the free-stream turbulence values.

Laminar separation bubbles can result from laminar separation followed by sufficiently early transition in the separated shear layer and subsequent turbulent reattachment. Errors in predicting the length of these bubbles will lead to failures in the blade design and wrong solutions. Early attempts at describing bubble development and separation, see [107, 108], were based on semi-empirical models. In those models, constant pressure for the region of the separated laminar shear layer, instantaneous transition, and linear variations in free-stream velocities during the phase of turbulent reattachment was assumed. An integral boundary layer computation procedure was applied, the location of the transition onset was found with correlations for separated laminar layer, in function of the momentum thickness and Re.

Flows with transition and separation phenomena could be modeled with application of contemporary models for accurate description of all aerodynamic effects, which are expected to be observed, together with innovative and very correct transition model. Modeling of bubble dynamics is important for the purposes of research and prediction of separated flows with transition [63].

In [109-113] various predictive techniques were described in detail in the area of turbulence in turbines and stressed on the need for application of improved and correct transition modeling.

In the literature, many papers discuss experiments and their outcomes related to turbine blades. Mostly, they present research in more global aspect, a small number of experiments provide detailed results useful for turbulence modeling. This is related to the fact that it is very difficult to obtain sufficiently thick boundary layers to perform detailed measurements on the suction and pressure sides. Many results are obtained after modeling and measurements related to research on a flat plate with a pressure gradient, imposed by the external wall [70, 114, 115] for negative pressure gradients; also, after application of Görtler vortex on the concave plate [116]. Results for the streaming effects of blade convex side, are shown in [12]. Studies in [117] discuss results of measurements on the suction surface of blade under conditions of very low Reynolds number.

There are mainly two approaches used to model bypass transition in industry [65]. The first is to apply low-Reynolds number turbulence models in which wall-damping functions implemented into the turbulent transport equations were applied to obtain the moment when boundary layer transition will occur [129, 130]. Research activities have proved that this approach cannot predict very well the influence of various factors, such as pressure gradients, free-stream turbulence, and wall roughness to predict the transition onset [131]. Damping functions, optimized to damp the turbulence in the viscous sublayer, cannot give reliable prediction of the transition when subjected different and complicated processes [132].

The second approach is application of experimental correlations related to the free-stream turbulence intensity and to the transition Reynolds number, with included boundary layer momentum thickness $[65,118]$. The last approach is proved as accurate, but very challenging, - actual momentum-thickness, Reynolds numbers must be compared with their critical values, obtained from correlations, included into the mathematical model, applied for the 
purposes to arrive to physically correct numerical solution. There are additional difficulties related to application of unstructured mesh, not well-defined boundary layer, and various approaches to attain numerical solution [64].

\section{An example of modeling of turbine stage with twisted rotor blade with three different turbulence models}

The main target of this research in which geometry modeling, numerical set-up, and convergence problem solution are described in detail in $[119,120]$ is to define the flow parameters distribution in a $3 \mathrm{D}$ turbine stage with twisted rotor blade. For the purposes of the turbulence modeling, the standard $k-\varepsilon$ turbulence model, RNG $k-\varepsilon$, standard $k-\varepsilon$, for the case of research on radial gap, and RSM (Reynolds stress model) models are applied, in regard to the flow conditions.

The Reynolds stress model (RSM) is applicable for modeling effects of additional vortices, found in flow and shear stress effects over fluid particles [122].

The standard $k-\varepsilon$ model gives quite good values, especially for the turbulent kinetic energy, in the core flow see $[119,121,122]$. In [123], results show that the advantage of using the RSM in regions of flow separation; however, the main flow features were still good enough, captured by the $k-\varepsilon$ model.

The RNG model gives the highest prediction of lift and maximal lift angle [124, 127].

The $k-\varepsilon$ turbulence models are appropriate for flows characterized by high adverse pressure and intensive separation. This model allows for a more accurate near wall treatment with an automatic switch from wall function to low-Reynolds number formulation, based on grid spacing see [125-128].

In the current study, it is found that depending on the specific flow feature, under consideration, different turbulence model have to be applied.

Qualitative results are shown in Figure 6-Figure 9.

Figure 6 shows velocity field distribution in the case of rotating rotor blade and activated the standard $k-\varepsilon$ turbulence model.

Figure 7 presents vorticity magnitude values, in radial direction, for the turbine stage under consideration.

Numerical results for pressure distribution, in the case of applied standard $k-\varepsilon$ model, are shown in Figure 8.

Figure 9 visualized vortices, in radial direction, due to difference between the pressure field values for hub and shroud sections in the turbine stage, in the case of applied Reynolds stress model (RSM). The area occupied by this vortex is bigger than the one formed in the case of standard $k-\varepsilon$ turbulence model, Figure 7 . 

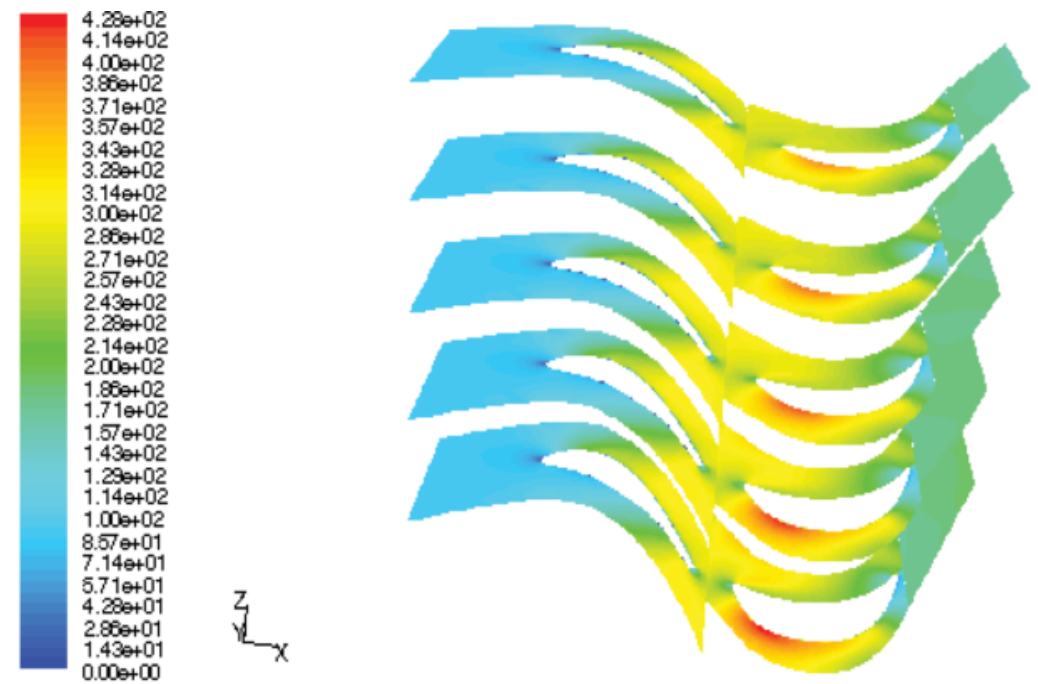

Conburs of Velocity Magnitude (m/s)

Figure 6. Flow velocity field distribution in control sections, in radial direction.
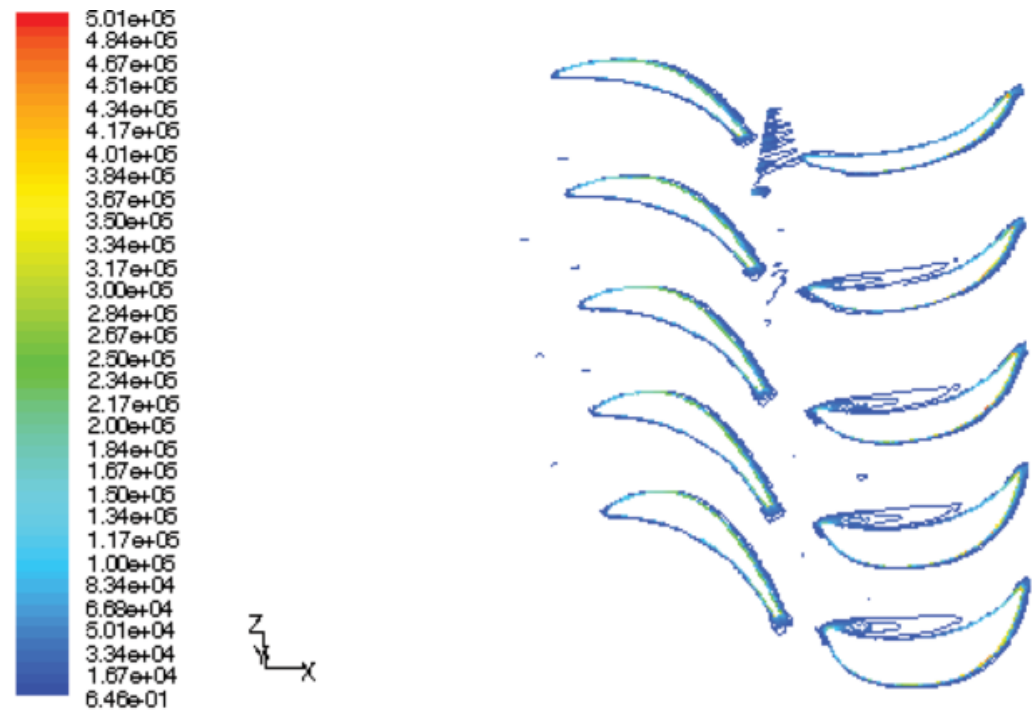

\section{Conburs of Vorlicity Magnitude (1/s)}

Figure 7. Vorticity magnitude values in control sections, in radial direction. 

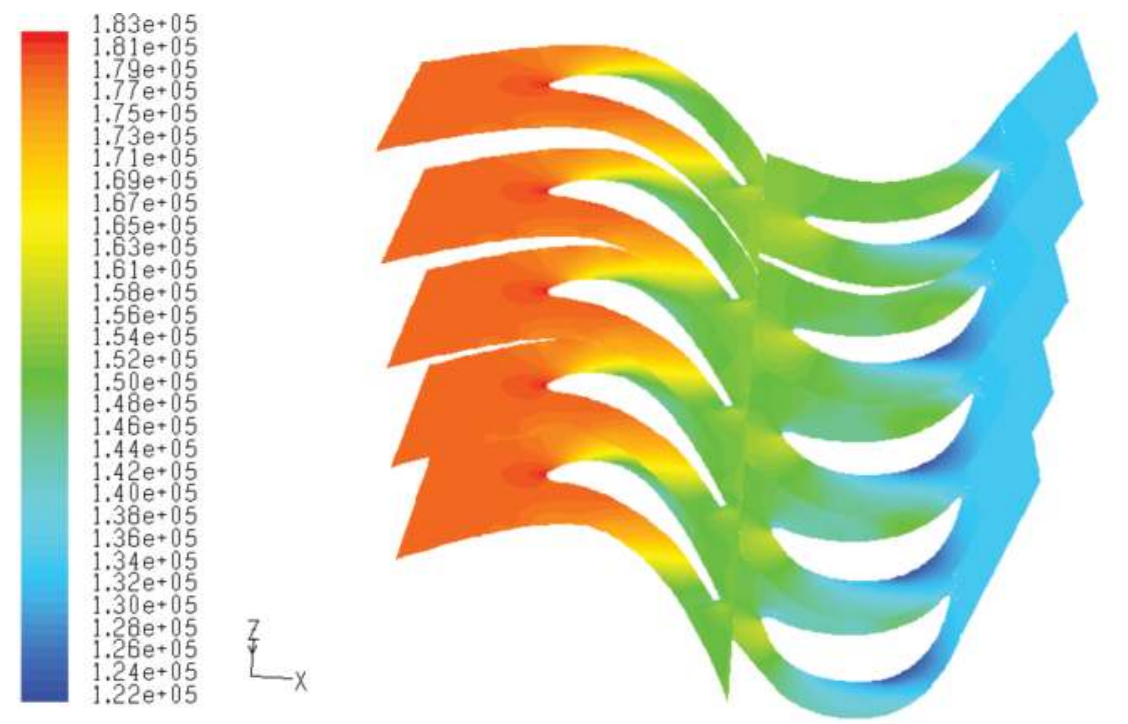

Contours of Static Pressure (pascal)

(a)

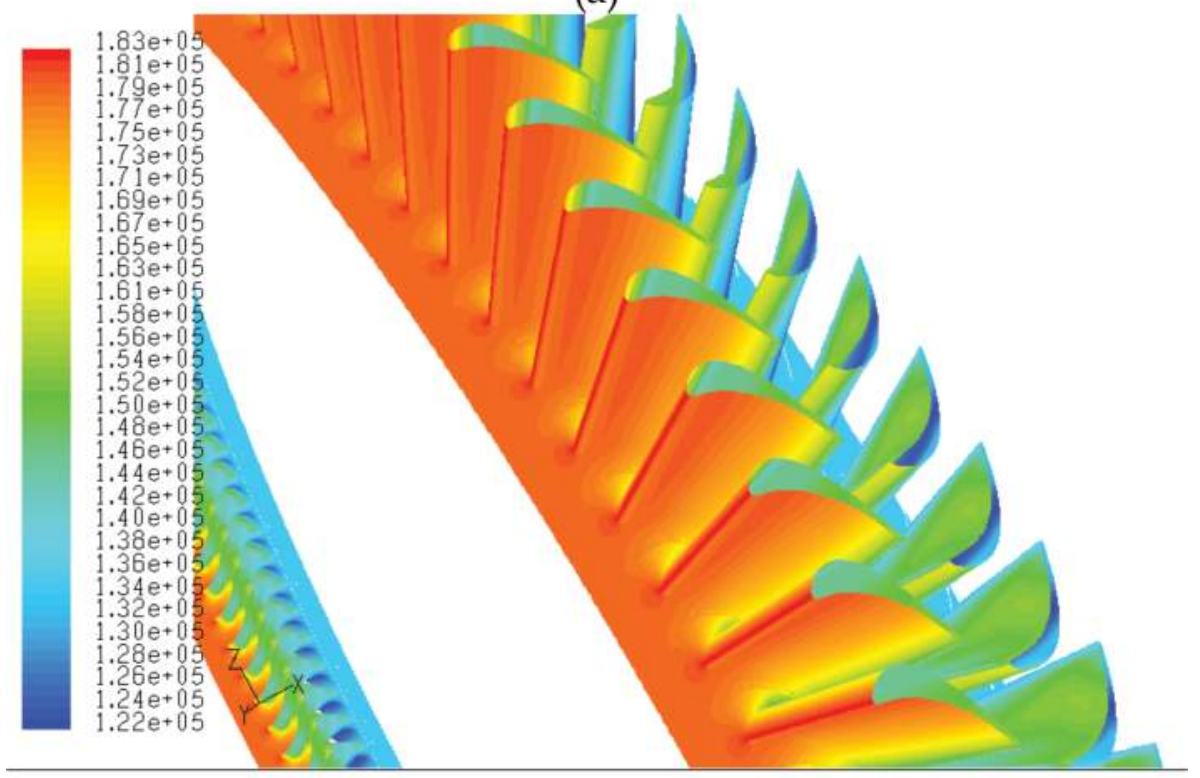

Contours of Static Pressure (pascal)

(b)

Figure 8. Static pressure distribution - by control sections (a), for the turbine blade (b) in the case of rotating rotor blade. 


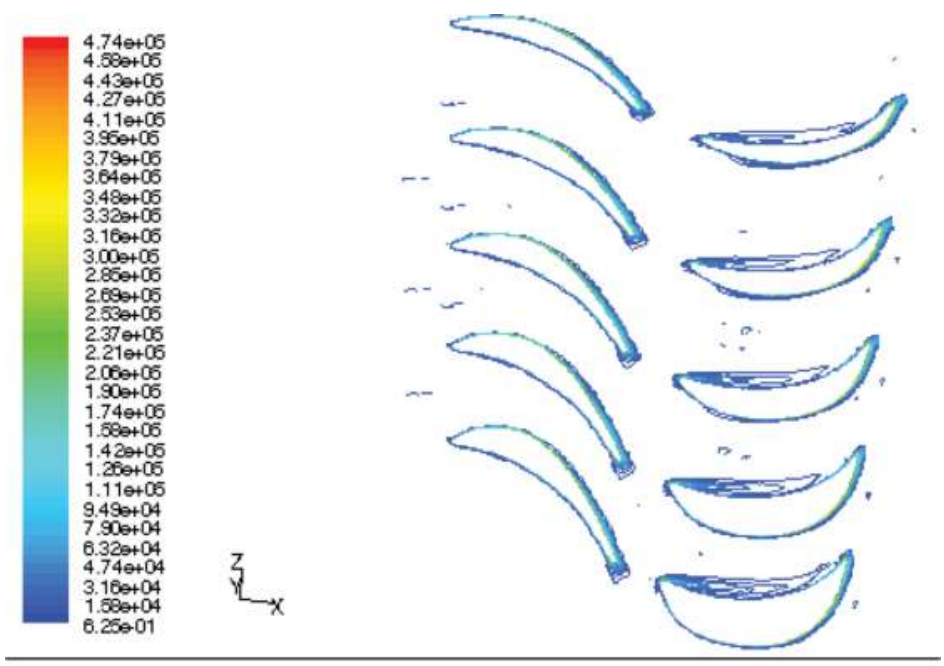

Conburs of Vorlicity Magnitude (1/s)

Figure 9. Vorticity magnitude values by control sections, in radial direction.

The outcomes of the performed research are as follows:

- The RNG model is acceptable to study both the shear stress and streamlines curvature effects. It presents vortices formed at the trailing edge and also provides results for aerodynamic features at the leading edge.

- In the case of applied RSM model, a relative decrease of $1.308 \%$ for turbine stage efficiency is observed. This is a result of taking into account of all pulsations and vortex structures near the wall regions, boundary layer separation, viscosity, and compressibility effects.

- The RNG $k-\varepsilon$ turbulence model leads to increased values for turbulent intensity and less turbulent viscosity. This is a prerequisite for decrease of the left-hand side term values in the momentum equations, furthermore causes relative increase of stage efficiency with $0.147 \%$, in a comparison with the case of implemented RSM turbulence model.

\section{Conclusion}

On the basis of previously performed numerical and experimental works by many researchers and by the author, transition onset, and turbulence origin, their effects and impact on efficiency, flow parameters distribution, and possible blades design. Various modeling techniques, turbulence models and their application are discussed to obtain physically correct prediction of turbulence effects in flow past turbine blades. Various turbulence features, and fluid dynamics specifics, streaming of blades and their efficiency performance are discussed. 
The chapter presents contemporary approaches to turbulence modeling and the adequacy of turbulence models to obtain flow characteristics, also.

This work could be very helpful for engineers working on prediction of transition onset, turbulence effects and their impact on the overall turbine performance. Moreover, it could be a basis for future research works related to innovative models and advanced numerical techniques to turbulence modeling and analysis.

\section{Acknowledgements}

This research is supported by the National Science Fund of Bulgaria and the Technical University - Varna.

\section{Author details}

Galina Ilieva Ilieva

Address all correspondence to: galinaili@yahoo.com

Technical University, Varna, Bulgaria

\section{References}

[1] McDonough JM. Introductory Lecturers on Turbulence, Physics, Mathematics and Modeling. Lexington, KY: Departments of Mechanical Engineering and Mathematics University of Kentucky. 2004/2007.

[2] Bingham R. Plasma physics: On the crest of a wake. Nature. 2007;445:721-722. http:// www.nature.com/nature/journal/v445/n7129/fig_tab/445721a_F1.html

[3] http://wavelets.ens.fr/RESULTATS/GAELE/Website/resume_anglais.html

[4] Denton JD. Loss mechanisms in turbomachines. IGTI Gas Turbine Scholar Lecture. 1993; ASME 93-GT-435.

[5] Dawes WN. A comparison of Zero and One Equation Turbulence Models for Turbomachinery calculations. ASME Paper No. 90-GT-303. 1990.

[6] Schlichting H. Boundary Layer Theory. 6th ed. New York: McGraw-Hill; 1966.

[7] Megerle, B. Unsteady aerodynamics of low pressure steam turbines operating under low volume flow conditions. These N 6096 (2014). École Polythechnique Federale de Lausanne, presented on 16 May 2014. 
[8] Raj R, Lakshminarayana B. Three-dimensional characteristics of turbulent wakes behind rotors of axial flow turbomachinery. ASME Journal of Engineering for Power. April 1976; 218-228.

[9] Chaluvadi VSP. Blade Vortex Interactions in High Pressure Steam Turbines. Girton College Department of Engineering University of Cambridge; November 2000.

[10] Binder A. Turbulent production due to secondary vortex cutting in a turbine rotor. Journal of Engineering for Gas Turbines and Power. 1985;107:1039-1046.

[11] Binder A, Forster W, Mach K, Rogge H. Unsteady flow interaction caused by stator secondary vortices in a turbine rotor. ASME Paper No. 86-GT-302; 1986.

[12] Sharma OP, Wells RA, Schlinker RH, Bailey DA. Boundary layer development on turbine airfoil suction surfaces. ASME Journal of Engineering for Power 1982;104:698-706.

[13] Harrison S. The Influence of Blade Stacking on Turbine Losses [Ph.D. thesis]. Cambridge University; 1989.

[14] Hawthorne WR. Rotational flow through cascades. Journal of Mechanics and Applied Mathematics. 1955; 8.

[15] Adamczyk JJ. Wake Mixing in Axial Flow Compressors. ASME Paper No. 96-GT-029; 1996.

[16] Van de Wall A, Kadambi JR, Adamczyk JJ. A Transport Model for the Deterministic Stresses Associated with Turbomachinery Blade Row Interactions. ASME Paper No. 2000-GT-430; 2000.

[17] Meyer RX. The Effect of Wakes on the Transient Pressure and Velocity Distributions in Turbomachines. ASME Journal of Basic Engineering. 1958;1544-1552.

[18] Kemp NH, Sears WR. The Unsteady Forces due to Viscous Wakes in Turbomachinery. Journal of Aeronautical Sciences. 1955;22(7):478-483.

[19] Smith LH. Wake Dispersion in Turbomachines. ASME Journal of Basic Engineering. 1966; 688-690.

[20] Hodson HP, Dawes WN. On the Interaction of Measured Profile Losses in Unsteady Wake-Turbine Blade Interaction Studies. ASME 96-GT-494, 1996.

[21] Deregel P, Tan CS. Impact of Rotor Wakes on Steady State Axial Compressor Performance. ASME Paper No. 96-GT-253; 1996.

[22] Valkov T. Control of the Unsteady Flow in a Stator Blade Row Interacting with Upstream Moving Wakes. ASME Journal of Turbomachinery. 1995;117:97-105.

[23] Bindon JP. Exit Plane and Suction Surface Flows in an Annular Cascade with a Skewed Inlet Boundary Layer. International Journal of Heat and Fluid Flow. 1980;2(2P):57.

[24] Boletis E, Sieverding CH, van Hove W. Effect of Skewed Inlet Endwall Boundary Layer on the 3-D Flowfield in an Annular Cascade, Viscous Effects in Turbomachinery. AGARD-CP-351; 1983. 
[25] Walsh JA, Gregory-Smith, DG. The Effect of Inlet Skew on the Secondary Flows and Losses in a Turbine Cascade, Efficiency Prediction Improvement. I Mech. E, Paper No. C275/87; 1987.

[26] Wadia AR, Booth, TC. Rotor-tip leakage: Part II - design optimisation through viscous analysis and experiment. ASME Journal of Engineering for Power. 1982;104:162.

[27] Sjolander SJ, Amrud KK. Effects of tip clearance on blade loading in a planar cascade of turbine blades, ASME Journal of Turbomachinery. 1987;109:237.

[28] Heyes FJG, Hodson HP, Dailey GM. The effect of blade tip geometry on the tip leakage flow in axial turbine cascades. ASME Journal of Turbomachinery. 1992;114:643.

[29] Moore J, Tilton JS. Tip leakage flow in a linear turbine cascade. ASME Journal of Turbomachinery. 1988; 110:18.

[30] Bindon JP. The measurement and formation of tip clearance loss. ASME Journal of Turbomachinery. 1989;111:257.

[31] Yaras MI, Sjolander SA. Prediction of Tip Leakage Losses in Axial Turbines. ASME Paper No. 90-GT-154; 1990.

[32] Atkin CJ, Squire LC. A study of the interaction of a normal shock wave with a turbulent boundary layer at mach numbers between 1.3 and $1.55^{\prime}$. European Journal of B/Fluids. 1992;11(1).

[33] Scherbakov AP, Tischenko AA, Dohler SW. The loss behaviour of transonic flow in nozzle cascades under the influence of unsteady effects. In: 2nd European Conference on Turbomachinery, Fluid Dynamics and Thermodynamics, Antwerpen; 5-7March 1997.

[34] Hetsroni G. Particles-turbulence interaction. International Journal of Multiphase Flow. 1989;15(5):735-746.

[35] Azzopardi BJ, Teixeira JCF. Detailed measurements of vertical annular two-phase flow Part It: gas core turbulence. ASME Journal of Fluids Engineering. 1994b;116: 796-800.

[36] Ilieva GI. Erosion failure mechanisms in turbine stage with twisted rotor blade. Engineering Failure Analysis. 2016;70: 90-104.

[37] Siddique W. Design of internal cooling passages: investigation of thermal performance of serpentine passages [Doctoral Thesis]. Stockholm, Sweden: Division of Heat and Power Technology, Department of Energy Technology, Royal Institute of Technology ; 2011.

[38] Simonich J, Bradshaw, P. Effect of free-stream turbulence on heat transfer through a turbulent boundary layer. Journal of Heat Transfer. 1978;100: 671-677.

[39] Blair M, Werle M. The Influence of Free-Stream Turbulence on the Zero Pressure Gradient Fully Turbulent Boundary Layer. (R80-914388-12), Contract No. F49620-78-C-0064. UTCRC, East Hartford, CT; 1980.

[40] Blair M. Influence of free-stream turbulence on turbulent boundary layer heat transfer and mean profile development, parts I and II. Journal of Heat Transfer. 1983;105 (1):33-47. 
[41] Hancock P. Effect of high free-stream turbulence in turbulent boundary layer [Ph.D. thesis]. UK: Imperial College, London University.

[42] Maciejewski P, Moffat R. Heat transfer with very high free-stream turbulence. Part I. Experimental Data. Journal of Heat Transfer. 1992;114 (4): 827-833.

[43] Du H, Ekkad S, Han J. Effect of unsteady wake with trailing edge coolant ejection on film cooling performance for a gas turbine blade. Journal of Turbomachinery. 1999;121 (3): 448-455.

[44] Du H, Han J, Ekkad S. Effect of unsteady wake on detailed heat transfer coefficient and film effectiveness distributions for a gas turbine blade. Journal of Turbomachinery. 1997;120 (4): 808-813.

[45] Du H, Han J, Ekkad SV. Effect of unsteady wake on detailed heat transfer coefficient and film effectiveness distributions for a gas turbine blade. Journal of Turbomachinery. 1998;120 (4): 808-817.

[46] Włodzimierz W. Numerical evaluation of the blade cooling for the supercritical steam turbine. Applied Thermal Engineering. 2013; 51(1): 953-962.

[47] Pietrzyk J, Bogard D, Crawford M. Hydrodynamic measurements of jets in crossflow for gas turbine film cooling applications. Journal of Turbomachinery. 1989;111 (2):139-145.

[48] Andreopoulos J. On the structure of jets in a crossflow. Journal of Fluid Mechanics. 1985;157:163-197.

[49] Jumper G, Elrod W, Rivir R. Film cooling effectiveness in high turbulence flow. Journal of Turbomachinery. 1991;113 (3): 479-483.

[50] Kadotani K, Goldstein R. On the nature of jets entering a turbulent flow: Part B - film cooling performance. Journal of Engineering for Power. 1979c;101 (3): 466-470.

[51] Bons J, MacArthur C, Rivir R. The effect of high free-stream turbulence on film cooling effectiveness. Journal of Turbomachinery. 1996;118(4): 814-825.

[52] Kodzwa Jr. PM, Eaton JK. Measurements of film cooling performance in a transonic single passage model. Report No. TF 93 June 2005, Flow Physics and Computation Division Department of Mechanical Engineering Stanford University Stanford, CA 94305-3035; 2005.

[53] Mehendale A., Han J. Influence of High Mainstream Turbulence on Leading Edge Film Cooling Heat Transfer, Journal of Turbomachinery. 1992;114 (4), 707-715.

[54] Ou S, Mehendale A, Han J. Influence of high mainstream turbulence on leading edge film cooling heat transfer: effect of film hole location. Journal of Turbomachinery. 1992;114 (4):716-723.

[55] Ou S, Han J. Influence of high mainstream turbulence on leading edge film cooling heat transfer through two rows of inclined film slots. Journal of Turbomachinery. 1992;114 (4):724-733. 
[56] Zhao L, et al. Numerical simulation on impingement and film composite cooling of blade leading edge model for gas turbine. Applied Thermal Engineering. 2014.

[57] Saumweber C, Schulz A, Wittig S. Free-stream turbulence effects on film cooling with shaped holes. Journal of Turbomachinery. 2003;125 (1): 65-73.

[58] Yue-Tzu Y, Wei T-C, Wang Y-H. Numerical study of turbulent slot jet impingement cooling on a semicircular concave surface. International Journal of Heat and Mass Transfer. 2011;54(1): 482-489.

[59] Öztekin E. et al. Heat transfer in a turbulent slot jet flow impinging on concave surfaces. International Communications in Heat and Mass Transfer. 2013;44:77-82.

[60] Emmons HW. The Laminar-turbulent transition in a boundary layer: Part I. Journal of Aeronautical Science. 1951;18(7): 490-498.

[61] Schubauer GB, Klebanoff PS. Contributions on the mechanics of boundary-layer transition. NACA Tech. Note TN 3489; 1955.

[62] Walker GJ, Gostelow JP. Laminar-Turbulent Transition in Boundary Layers on Axial Turbomachine Blades. ERCOFTAC Bulletin. 2009;80:11-15.

[63] Jahanmiri M. Boundary Layer Transitional Flow in Gas Turbines, Research Report 2011:01, Göteborg, Sweden: Division of Fluid Dynamics Department of Applied Mechanics, Chalmers University of Technology; 2011.

[64] Reza Taghavi Z, Salary M, Kolaei A. Prediction of boundary layer transition based on modeling of laminar fluctuations using RANS approach. Chinese Journal of Aeronautics. 2009;22:113-120.

[65] Mayle RE. The role of laminar-turbulent transition in gas turbine engines. ASME Journal of Turbomachinery. 1991;113: 509-537.

[66] Sieverding CH. Recent Progress in the Understanding of Basic Aspects of Secondary Flows in Turbine Blade Passages, ASME Paper No. 84-GT-78; 1984.

[67] Gaster M. The structure and behavior of laminar separation bubbles. ARC R\&M Report No. 3595; 1969.

[68] Hodson HP. Boundary layer and loss measurements on the rotor of an axial-flow turbine. ASME Journal of Engineering for Gas Turbines and Power. 1984;106: 391-399.

[69] Warren JM, Metzger DE. Heat transfer with film cooling in the presence of laminarizing mainstream. ASME Paper 72-HT-11; 1972.

[70] Blair MF. Influence of free-stream turbulence on boundary layer transition in favorable pressure gradients. ASME Engineering for Gas Turbines and Power. 1982;104:743-750.

[71] Sharma OP. Momentum and thermal boundary layer development on turbine airfoil suction surfaces. AIAA Paper No. 87-1918;1987. 
[72] Taniguchi H, Sakai H, Funazaki K. Effects of free-stream turbulence on bypass transition of separated boundary layer on low-pressure turbine airfoils. In: Proceedings of 3rd Asian Joint Workshop on Thermophysics and Fluid Science, Matsue, Japan; 2010.

[73] Bons JP, McClain ST, Taylor RP, Rivir RB. The many faces of turbine surface roughness. ASME Journal of Turbomachinery. 2001;123:739-748.

[74] Taylor RP. Surface roughness measurements on gas turbine blades. ASME Journal of Turbomachinery. 1990;112:175-180.

[75] Kind RJ, Serjak PJ, Abbott MWP. Measurements and prediction of the effects of surface roughness on profile losses and deviation in a turbine cascade. ASME Journal of Turbomachinery. 1998;120:20-27.

[76] Boynton JL, Tabibzadeh R, Hudson ST. Investigation of rotor blade roughness effects on turbine performance. ASME Journal of Turbomachinery. 1992;115: 614-620.

[77] Suder KL, Chima RV, Strazisar AJ, Roberts WB. The effect of adding roughness and thickness to a transonic axial compressor rotor. ASME Journal of Turbomachinery. 1995;117: 491-505.

[78] Bons JP, McClain ST. The effect of real turbine roughness with pressure gradient on heat transfer. ASME Journal of Turbomachinery. 2003;126:333-441.

[79] Blair MF. An experimental study of heat transfer in a large scale turbine rotor passage. ASME Journal of Turbomachinery. 1994;116:1-13.

[80] Wang T, Rice MC. Effect of elevated free-stream turbulence on transitional heat transfer over dual-scaled rough surfaces. ASME GT2003-38835; 2003.

[81] Götler H. Über eine dreidimensionale Instabiltät laminar Grenzschichten an konkaven Wänden. Nachr. Wiss. Ges. Göttingen, Math Physics Klasse, Neue Folge 1940;2(1); see also ZAMM, 1941;21; 250-252.

[82] Liepmann HW. Investigations on laminar boundary layer stability and transition on curved boundaries. NACA ACR 3H30 (NACA-WR-W-107); 1943.

[83] Martin BW, Brown A. Factors influencing heat transfer to the pressure surfaces of gas turbine blades. International Journal of Heat and Fluid Flow. 1979;1:107-119.

[84] Morkovin MV. On the Many Faces of Transition. In: Wells CS, editor. Viscous Drag Reduction. New York: Plenum Press. 1969. pp. 1-31.

[85] Colban W, Gratton A, Thole KA, Haendler M. Heat transfer and film-cooling measurements on a stator vane with fan-shaped cooling holes. ASME Journal of Turbomachinery. 2006;128: 53-61.

[86] Colban W, Thole KA, Haendler M. Experimental and computational comparisons of fan-shaped film cooling on a turbine vane surface. ASME Journal of Turbomachinery. 2007;129:23-31. 
[87] Elsner W. Transition modelling in turbomachinery. Journal of Theoretical and Applied Mechanics. 2007;45(3):539-556.

[88] Elsner W, Vilmin S, Drobniak S, Piotrowski W. Experimental analysis and prediction of wake-induced transition in turbomachinery. ASME Paper no. GT2004-53757, ASME TURBO EXPO 2004, Vienna.

[89] http://documentslide.com/documents/1-intro-turbulent-flows.html

[90] Sharif MAR, Mothe KK. Evaluation of turbulence models in the prediction of heat transfer due to slot jet impingement numerical heat transfer, part B: Fundamentals. International Journal of Computation and Methodology, 2009;55:(4):273-294.

[91] Ali ARA, Janajreh I. Numerical simulation of turbine blade cooling via jet impingement. Energy Procedia. 2015;75: 3220-3229.

[92] Bredberg, J. Turbulence Modelling for Internal Cooling of Gas-Turbine Blades. Goteborg, Sweden: Department of Thermo and Fluid Dynamics, Chalmers University of Technology; 2002.

[93] Acharya S, Dutta S, Myrum TA, Baker RS. Periodically developed flow and heat transfer in a ribbed duct. International Journal of Heat and Mass Transfer. 1993;36:2069-2082.

[94] Isman MK, Pulat E, Etemoglu AB, Can M. Numerical investigation of turbulent impinging jet cooling of a constant heat flux surface. Numerical Heat Transfer, Part A. 2008;53:1109-1132.

[95] Simoneau RJ, Simon FF. Progress towards understanding and predicting heat transfer in the turbine gas path. International Journal of Heat Fluid Flow. 1992;14 (2):106-128.

[96] Stephens MA. Computation of flow and heat transfer in a rectangular channel with ribs. AIAA Paper 95-0180; 1995.

[97] Gatski TB, Speziale CG. On explicit algebraic stress models for complex turbulent flows. Journal of Fluid Mechanics. 1993;254:59-78.

[98] Speziale C. Comparison of explicit and traditional algebraic stress models of turbulence. AIAA Journal. 1997;35 (9):1506-1509.

[99] Iacovides H, Raisee M. Recent progress in the computation of flow and heat transfer in internal cooling passages of turbine blades. International Journal of Heat Fluid Flow. 1999;20:320-328.

[100] Durbin PA. Separated flow computations with the $k-f-v^{2}$ model. AIAA Journal. 1995; 33:659-664.

[101] Parneix S, Durbin PA, Behnia M. Computation of 3-D turbulent boundary layers using the $\mathrm{v}^{2}$-f model. Flow, Turbulence Combustion. 1998;60:19-46.

[102] Behnia M, Parneix S, Durbin P. Prediction of heat transfer in an axisymmetric turbulent jet impinging on a flat plate. International Journal of Heat Mass Transfer. 1998;41:1845-1855. 
[103] Behnia M, Parneix S, Shabany Y, Durbin PA. Numerical study of turbulent heat transfer in confined and unconfined impinging jets. International Journal of Heat Fluid Flow. 1999;20:1-9.

[104] Metzger DE, Bunker RS, Chyu RK. Cavity heat transfer on a transverse grooved wall in a narrow flow channel. Journal of Heat Transfer. 1989;111:73-79.

[105] Spalart PR, Allmaras SR. A one-equation turbulence model for aerodynamic flows. AIAA;92-0439; 1992.

[106] Bardina JE, Huang PG, Coakley TJ. Turbulence modeling validation, testing, and development. NASA TM110446; 1997.

[107] Horton HP. A Semi-Empirical Theory for the Growth and Bursting of Laminar Separation Bubbles. ARC CP 1073; 1969.

[108] Roberts WB. Calculation of laminar separation bubbles and their effects on airfoil performances. AIAA Journal. 1980;18: 25-31.

[109] Lakshminarayana B. An assessment of computational fluid dynamic techniques in the analysis and design of turbomachinery: - The 1990 freeman scholar lecture. ASME Journal of Fluids Engineering. 1991;113: 315-352.

[110] Simoneau RJ, Simon FF. Progress towards understanding and predicting heat transfer in the turbine gas path. International Journal of Heat Fluid Flow. 1993;14:106-128.

[111] Simon FF, Ashpis DE. Progress in Modeling of Laminar to Turbulent Transition on Turbine Vanes and Blades. NASA Technical Memorandum No. 107180; 1996.

[112] Dunn MG. Convective Heat Transfer and Aerodynamics in Axial Flow Turbines. ASME Paper No. 2001-GT-0506; 2001.

[113] Yaras MI. Measurements of the Effects of Freestream Turbulence on Separation Bubble Transition. ASME Paper No. GT-2002-30232; 2002.

[114] Keller FJ, Wang T. Flow and Heat Transfer Behavior in Transitional Boundary Layers with Streamwise Acceleration. ASME 94-GT-24; 1994.

[115] Kestoras MD, Simon TW. Effect of Free-Stream Turbulence Intensity on a Boundary Layer Recovering from Concave Curvature Effects. ASME 93-GT-25; 1993.

[116] Peerhossaini, H, Wesfreid JE. Experimental study of the Görtler-Taylor instability. In: Wesfreid JE, Brand H, Mannville P, Albinet G, Boccara N, editors. Propagation in Systems for From Equilibrium. Berlin: Springer; 1988. pp. 399-412.

[117] van Treuren KW, Simon T, von Koller M, Byerley AR, Baughn JW, Rivir R. Measurements in a turbine cascade flow under ultra low Reynolds number conditions. Journal of Turbomachinery, Transaction of ASME. 2002;124:100-106.

[118] Abu-Ghannam BJ, Shaw R. Natural transition of boundary layers-the effects of turbulence, pressure gradient, and flow history. Journal of Mechanical Engineering Science. 1980;22(5):213-228. 
[119] Ilieva GI. Modelling, Research and Analysis of 3D Real Flow in Turbine Stages with Complex Geometry [Dissertation Thesis]. Varna: Technical University, 2009.

[120] Ilieva G. Numerical Modeling and Research of 3D Turbine Stage. Engineering Applications of Computational Fluid Dynamics. (Series of Advanced Structured Materials) 2015;44:103-126.

[121] Buntić Ogor I, Dietze S, Ruprecht A. Numerical Simulation of the Flow in Turbine-99 Draft Tube. In: Proceedings of the third IAHR/ERCOFTAC Workshop on Draft Tube Flow; 8-9 December 2005; Porjus, Sweden.

[122] Menter F. Turbulence Modeling for Turbomachinery Applications, Lucerne: QNETCFD Meeting; 2002.

[123] Villalpando F, Reggio M, Ilinca A. Assessment of turbulence models for flow simulation around a wind turbine airfoil. Modelling and Simulation in Engineering. 2011;2011, Article ID 714146, 8 pages http://dx.doi.org/10.1155/2011/714146

[124] Ilieva GI, Iosifov RD. Geometry modeling features for 3D turbine cascade with twisted rotor blade in GAMBIT. Acta Universitatis Pontica Euxinus. 2005;5(2):7-12 2005; ISSN 1312-1669.

[125] Menter FR. Two-equation eddy-viscosity turbulence models for engineering applications. AIAA Journal. 1994;32 (8):1598-1605.

[126] Menter FR. Zonal Two Equation k-w Turbulence Models for Aerodynamic Flows. AIAA Paper \#93-2906, 24th Fluid Dynamics Conference; July 1993.

[127] Jošt D, et al. Numerical flow simulation and efficiency prediction for axialturbines by advanced turbulence models. In 2012 IOP Conference Series: Earth and Environmental Science. 2012. 15062016 doi:10.1088/1755-1315/15/6/062016

[128] Cleak JGE, Gregory-Smith DG. Turbulence modeling for secondary flow prediction in a turbine cascade. Journal of Turbomachinery. 1992;114(3):590-598. doi:10.1115/1.2929183

[129] Savill AM. Some Recent Progress in the Turbulence Modelling of Bypass Transition. In: So RMC, Speziale CG, Launder BE, editors. Near-Wall Turbulent Flows. Elsevier Science Publishers B.V.; 1993. pp. 829-848.

[130] Savill AM. One-Point Closures Applied to Transition: Turbulence and Transition Modelling. Dordrecht: Kluwer Academic Publisher; 1996. pp. 233-268.

[131] Suzen YB, Huang PG, Hultgren, LS, et al. Predictions of separated and transitional boundary layers under low-pressure turbine airfoil conditions using an intermittency transport equation. Journal of Turbomachinery. 2003:125(3):455-464.

[132] Reynolds O. An experimental investigation of the circumstances which determine whether the motion of water shall be direct or sinuous, and of the law of resistance in parallel channels. Philosophical Transactions of the Royal Society of London A. 1883;174:935-982. 
